\title{
Generalized Identifiability Conditions for Blind Convolutive MIMO Separation
}

\author{
Marc Castella*, and Eric Moreau ${ }^{\dagger}$, Senior Member, IEEE \\ * Institut Télécom; Télécom \& Management Sudparis; UMR-CNRS 5157 \\ 9 rue Charles Fourier, 91011 Évry Cedex, France \\ Email: marc.castella@it-sudparis.eu_ Fax: (+33) 160764433 \\ $\dagger$ University of Sud Toulon Var, ISITV, LSEET UMR-CNRS 6017 \\ av. G. Pompidou, BP56, F-83162 La Valette du Var Cedex, France \\ Email: moreau@univ-tln.fr \\ Fax: (+33) 494142448
}

\begin{abstract}
This paper deals with the problem of source separation in the case where the output of a multivariate convolutive mixture is observed: we propose novel and generalized conditions for the blind identifiability of a separating system. The results are based on higher-order statistics and are valid in the case of stationary but not necessarily i.i.d. signals. In particular, we extend recent results based on second-order statistics only. The approach relies on the use of so called reference signals. Our new results also show that only weak conditions are required on the reference signals: this is illustrated by simulations and opens up the possibility of developing new methods.
\end{abstract}

Index Terms-Higher order statistics, MIMO convolutive mixtures, Blind source separation, MIMO identification, Contrast functions, Independent Component Analysis, Reference system, Semi-blind methods

\section{INTRODUCTION}

The problem of blind source separation has a very large scope of potential applications such as telecommunications, array processing, audio processing and biology. This explains a high interest in the field over the last decades. Numerous achievements have already been accomplished through the now well-recognized concept of Independent Component Analysis (ICA), see e.g. [8]. The first extensively studied mixture model was the instantaneous one. However, more recently, the more general convolutive model has been considered [1], [9], [14] and successful algorithms have been proposed [16], [18]. In particular, this has been done through the derivation of so called contrast functions. Such functions are very useful since they provide both identifiability conditions and criteria to be optimized. Classically they are based on statistics of order higher than or equal to three, which may lead to complicated optimization schemes. To simplify the optimization step, further improvements, which are based on the idea of reference signals, have been recently introduced [4], [6], [7], [12], [13]. In a semi-blind context, the reference signals can be interpreted as a partial knowledge on the sources: this has not been fully exploited up to now and it is done in our paper.

Two practical ways to consider the problem of blind identifiability can be found. The first one uses second order statistics

Copyright (c) 2008 IEEE. Personal use of this matrerial is permitted. However, permission to use this material for any other purposes must be obtained from the IEEE by sending a request to pubs-permissions@ieee.org. through matrix algebra decompositions, see e.g. [10], [11], [17]. The second one uses high order statistics (higher than three) through contrast functions, see e.g. [9], [14]-[16]. In all cases, questions about identifiability conditions remain unclear and particularly the link between the above two points of view. To a certain extent, the use of reference signals in conjunction with higher order statistics makes a bridge between different statistics. This is what we extensively use in the following.

In this paper, the following main novel points are introduced:

- We provide generalized conditions for the identifiability of a separating system, which include previous conditions as well as new ones (e.g. spatially dependent sources,...).

- We propose new results which make a clear link between the ones in [7], [13], which consider contrast functions, and the one in [11], which consider second order statistics.

- We show that the so-called reference signals can be used in a semi-blind context under weak condition. The corresponding simulation results legitimate the interest of our new approach.

The paper is organized as follows. Section II states the problem; it also introduces the notations and proves preliminary results. The main results are then given in Section III. Section IV details situations where the former results hold and the simulations illustrate the case of a semi-blind context. Further interpretations and links with former works are detailed in Section V. Finally, Section VI concludes the paper.

\section{PRoblem STATEMENT AND PRELIMINARIES}

\section{A. Mixing model and notations}

In the whole paper, we consider discrete-time signals which by convention depend on a time index $n \in \mathbb{Z}$ ( $n$ hence stands for a generic integer). For any positive integer $N$, we denote by $\mathcal{I}_{N}$ the set $\{1, \ldots, N\}$. We consider the following multichannel convolutive mixing model:

$$
\mathbf{x}(n)=\sum_{k \in \mathbb{Z}} \mathbf{M}(k) \mathbf{s}(n-k),
$$

where $\mathbf{s}(n) \triangleq\left(s_{1}(n), \ldots, s_{N}(n)\right)^{\mathrm{T}}$ is the $N$-dimensional random source vector, $\mathbf{x}(n) \triangleq\left(x_{1}(n), \ldots, x_{Q}(n)\right)^{\mathrm{T}}$ is the 
$Q$-dimensional output vector of observations, and $\mathbf{M}(n)=$ $\left(M_{i j}(n)\right)_{(i, j) \in \mathcal{I}_{Q} \times \mathcal{I}_{N}}$ is the $Q \times N$ matrix of impulse responses of the mixing MIMO channel. We also consider the so-called multichannel separating system whose action on the observations reads:

$$
\mathbf{y}(n)=\sum_{k \in \mathbb{Z}} \mathbf{W}(k) \mathbf{x}(n-k) .
$$

In the latter relation $\mathbf{y}(n) \triangleq\left(y_{1}(n), \ldots, y_{N}(n)\right)^{\mathrm{T}}$ is the $N$ dimensional output of the separating system and $\mathbf{W}(n)=$ $\left(W_{i j}(n)\right)_{(i, j) \in \mathcal{I}_{N} \times \mathcal{I}_{Q}}$ is the $N \times Q$ matrix of impulse responses of the separating channel. For the sake of clarity, the signals, the mixing and the separating systems are assumed to be real-valued. The results can nevertheless be extended to the complex case. Additionally, we introduce the combined mixing-separating system defined by the impulse response $\mathbf{G}(n) \triangleq \sum_{k \in \mathbb{Z}} \mathbf{W}(n-k) \mathbf{M}(k)$. We thus have the following relation:

$$
\mathbf{y}(n)=\sum_{k \in \mathbb{Z}} \mathbf{G}(k) \mathbf{s}(n-k) .
$$

The objective of source separation consists in recovering $\mathbf{s}(n)$ from the observations $\mathbf{x}(n)$. In other words the separating system is searched for, such that $\mathbf{y}(n)$ corresponds to an estimation of $\mathbf{s}(n)$. When only the observations are used, but no information on the mixing system, the problem is referred to as the blind source separation problem. Any situation where little and incomplete information is exploited in addition to $\mathbf{x}(n)$ can be referred to as a semi-blind approach. The problem is tightly connected with the one of blind identification of the mixing system or of a separating system. In all cases, assumptions are required on the source signals and the mixing system. For the derivations in this paper more specifically, we need to introduce an $(R-2)$-dimensional vector $\mathbf{r}(n)$ with components $r_{j}(n), j \in \mathcal{I}_{R-2}$, where $R \geq 2$ is a given integer. These signals are called "reference signals" [4], [7], [13] and they can be used for the separation. As explained in [4], [7], these references may come from an additional information or may be constructed from the observations. We now sum up the necessary assumptions which hold throughout the paper:

A0. (i) The source signals $s_{i}(n), i \in \mathcal{I}_{N}$ are zero mean and unit variance.

(ii) The mixing system is stable and left-invertible.

(iii) The signals $r_{j}(n), j \in \mathcal{I}_{R-2}$ and $s_{j}(n), j \in \mathcal{I}_{N}$ are jointly stationary up to the $R$-th order under consideration.

The first assumption can be made with no loss of generality, whereas the second one is obviously a necessary one to invert the mixing system. The third assumptions restrict ourselves to stationary signals and systems.

Surprisingly, we do not require the common assumption of spatial independence of the source signals. A corresponding, but weaker assumption will indeed appear later when necessary: thus, our general approach will include results which showed that independence is sometimes not required [2]. Finally, one should notice that the derivations in the present paper do not require that the signals be temporally independent and identically distributed (i.i.d.). They remain valid in the general non i.i.d. case.

\section{B. Higher order statistics and cumulant decompositions}

The identifiability conditions derived in this paper specify the conditions to be met by the vector-valued reference signal $\mathbf{r}(n)$ when it is used in higher order statistics. In addition, note that derivations similar to those in this section can be carried out in the case $R=2$ (see Section III-A). We consider higher order cumulants and define the following $R$-th order crosscumulant:

$$
\begin{aligned}
& C_{s_{i_{1}} s_{i_{2}}}^{\mathbf{r}}(\mathbf{n})= \\
& \operatorname{Cum}\left\{s_{i_{1}}(n), s_{i_{2}}\left(n-n_{1}\right), r_{1}\left(n-n_{2}\right), \ldots, r_{(R-2)}\left(n-n_{2}\right)\right\}
\end{aligned}
$$

Note that it exists and depends on $\mathbf{n}=\left(n_{1}, n_{2}\right)$ only according to part (iii) of assumption A0. We will write in detail $C_{s_{i_{1} s_{i_{2}}}}^{\mathbf{r}}\left(n_{1}, n_{2}\right)$ instead of $C_{s_{i_{1}} s_{i_{2}}}^{\mathbf{r}}(\mathbf{n})$ only when necessary.

We consider a multidimensional $z$-transform of order two w.r.t. the variables $\mathbf{n}=\left(n_{1}, n_{2}\right)$. It is defined by:

$$
C_{s_{i_{1}} s_{i_{2}}}^{\mathbf{r}}[\mathbf{z}]=\sum_{\mathbf{n} \in \mathbb{Z}^{2}} C_{s_{i_{1}} s_{i_{2}}}^{\mathbf{r}}(\mathbf{n}) z_{1}^{-n_{1}} z_{2}^{-n_{2}}
$$

where $\mathbf{z}=\left(z_{1}, z_{2}\right) \in\left(\mathbb{C}^{*}\right)^{2}$. For the signals $\mathbf{x}(n)$ and $\mathbf{y}(n)$, the cumulants $C_{x_{i_{1}} x_{i_{2}}}^{\mathbf{r}}(\mathbf{n}), C_{y_{i_{1}} y_{i_{2}}}^{\mathbf{r}}(\mathbf{n})$ and their respective $z$ transforms $C_{x_{i_{1}} x_{i_{2}}}^{\mathbf{r}}[\mathbf{z}], C_{y_{i_{1}} y_{i_{2}}}^{\mathbf{r}}[\mathbf{z}]$ are defined similarly to the definitions given by (4) and (5). The matrix transfer function $\mathbf{M}\left[z_{1}\right]$ of the mixing MIMO channel is given by the following $z$-transform

$$
\mathbf{M}\left[z_{1}\right]=\sum_{n \in \mathbb{Z}} \mathbf{M}(n) z_{1}^{-n} .
$$

A similar definition holds for the matrix transfer function $\mathbf{W}\left[z_{1}\right]$ of the separator and it is known that the matrix transfer function of the global system corresponding to (3) reads $\mathbf{G}\left[z_{1}\right]=\mathbf{W}\left[z_{1}\right] \mathbf{M}\left[z_{1}\right]$. Let us introduce the matrices $\mathbf{C}_{\mathbf{x}}^{\mathbf{r}}[\mathbf{z}]$ and $\mathbf{C}_{\mathbf{s}}^{\mathbf{r}}[\mathbf{z}]$ whose $(i, j)$ components are $C_{x_{i} x_{j}}^{\mathbf{r}}[\mathbf{z}]$ and $C_{s_{i} s_{j}}^{\mathbf{r}}[\mathbf{z}]$ respectively. The following proposition will be useful in section III.

Proposition 1: We have the following decomposition of matrix $\mathbf{C}_{\mathbf{x}}^{\mathbf{r}}[\mathbf{z}]$

$$
\mathbf{C}_{\mathbf{x}}^{\mathbf{r}}[\mathbf{z}]=\mathbf{M}\left[z_{1} z_{2}\right] \mathbf{C}_{\mathbf{s}}^{\mathbf{r}}[\mathbf{z}] \mathbf{M}\left[z_{1}^{-1}\right]^{\mathrm{T}}
$$

Proof: Using (1), we have component-wise $x_{i}(n)=$ $\sum_{j \in \mathcal{I}_{N}} \sum_{k \in \mathbb{Z}} M_{i j}(k) s_{j}(n-k)$ and thus, using the multilinearity property of cumulants,

$$
\begin{gathered}
C_{x_{i_{1}} x_{i_{2}}}^{\mathbf{r}}(\mathbf{n})= \\
\sum_{\mathbf{j} \in \mathcal{I}_{N}^{2}} \sum_{\mathbf{k} \in \mathbb{Z}^{2}} M_{i_{1} j_{1}}\left(k_{1}\right) M_{i_{2} j_{2}}\left(k_{2}\right) C_{s_{j_{1}} s_{j_{2}}}^{\mathbf{r}}\left(n_{1}+k_{2}-k_{1}, n_{2}-k_{1}\right) .
\end{gathered}
$$


Now using definition (5) for the signal $\mathbf{x}(n)$, we have

$$
\begin{aligned}
C_{x_{i_{1}} x_{i_{2}}}^{\mathbf{r}}[\mathbf{z}]= & \sum_{\mathbf{n} \in \mathbb{Z}^{2}} \sum_{\mathbf{j} \in \mathcal{I}_{N}^{2}} \sum_{\mathbf{k} \in \mathbb{Z}^{2}} M_{i_{1} j_{1}}\left(k_{1}\right) M_{i_{2} j_{2}}\left(k_{2}\right) \\
= & \sum_{\mathbf{n} \in \mathbb{Z}^{2}} \sum_{\mathbf{j} \in \mathcal{I}_{N}^{2}}^{\mathbf{r}} \sum_{\mathbf{k} \in \mathbb{Z}^{2}} M_{i_{1} j_{1}}\left(k_{1}\right) M_{i_{2} j_{2}}\left(k_{2}\right) \\
= & \left.\sum_{\mathbf{j} \in \mathcal{I}_{N}^{2}} \sum_{\mathbf{k} \in \mathbb{Z}^{2}}^{\mathbf{r}} M_{1}+k_{2}-k_{1}, n_{2}-k_{1}\right) z_{1}^{-n_{1}} z_{2}^{-n_{2}} \\
& C_{s_{j_{1}}}^{\mathbf{r}}{ }_{s_{j_{2}}}[\mathbf{z}] z_{1}^{k_{2}-k_{1}}\left(k_{1}\right) z_{2}^{-k_{1}} M_{i_{2} j_{2}}\left(k_{2}\right) \\
= & \sum_{\mathbf{j} \in \mathcal{I}_{N}^{2}} M_{i_{1} j_{1}}\left[z_{1} z_{2}\right] M_{i_{2} j_{2}}\left[z_{1}^{-1}\right] C_{s_{j_{1}} s_{j_{2}}}^{\mathbf{r}}[\mathbf{z}]
\end{aligned}
$$

Component-wise, this is the result given by (7) in the proposition.

\section{HIGHER-ORDER IDENTIFIABILITY CONDITIONS}

Based on the properties given in the previous section, we generalize in this section identifiability conditions of a FIR MIMO system. We first illustrate how second-order statistics appear as a specific case of the results stated above.

\section{A. Second-order statistics}

Second order statistics appear formally as a particular case of the preceding results when $R=2$. Equation (4) where there is no reference signal simply corresponds to the correlation for which we adopt the specific notation:

$$
\begin{aligned}
& \Gamma_{s_{i_{1}} s_{i_{2}}}\left(n_{1}\right) \triangleq \operatorname{Cum}\left\{s_{i_{1}}(n), s_{i_{2}}\left(n-n_{1}\right)\right\} \\
& \Gamma_{\mathbf{s}}\left(n_{1}\right)=\left(\Gamma_{s_{i_{1}} s_{i_{2}}}\left(n_{1}\right)\right)_{\left(i_{1}, i_{2}\right) \in \mathcal{I}_{N}^{2}}
\end{aligned}
$$

Corresponding to (5), we have the power spectral matrix of the sources defined by:

$$
\boldsymbol{\Gamma}_{\mathbf{s}}\left[z_{1}\right] \triangleq \sum_{n_{2} \in \mathbb{Z}} \boldsymbol{\Gamma}_{\mathbf{s}}\left(n_{1}\right) z_{1}^{-n_{1}}=\left(\Gamma_{s_{i_{1}} s_{i_{2}}}\left[z_{1}\right]\right)_{\left(i_{1}, i_{2}\right) \in \mathcal{I}_{N}^{2}}
$$

Note that contrary to (7), $z_{2}$ does not appear here. Similar notations hold for the observations $\mathbf{x}(n)\left(\boldsymbol{\Gamma}_{\mathbf{x}}\left(n_{1}\right)\right.$ and $\boldsymbol{\Gamma}_{\mathbf{x}}\left[z_{1}\right]$ respectively). Similarly to (7), we now have the well-known relation:

$$
\boldsymbol{\Gamma}_{\mathbf{x}}\left[z_{1}\right]=\mathbf{M}\left[z_{1}\right] \boldsymbol{\Gamma}_{\mathbf{s}}\left[z_{1}\right] \mathbf{M}\left[z_{1}^{-1}\right]^{\mathrm{T}}
$$

\section{B. Identifiability of one source}

For a given vector-valued reference signal $\mathbf{r}(n)$, we first explicit a condition on $\mathbf{C}_{\mathbf{s}}^{\mathbf{r}}[\mathbf{z}], i \in\{1, \ldots, N\}$ to be able to identify one source of the mixture. The following proposition sums up our main result:

Proposition 2: Assume that

A1. the matrices $\boldsymbol{\Gamma}_{\mathbf{s}}\left[z_{1}\right]$ and $\mathbf{C}_{\mathbf{s}}^{\mathbf{r}}[\mathbf{z}]$ are diagonal,

A2. there exist a diagonal element of $\mathbf{C}_{\mathbf{s}}^{\mathbf{r}}[\mathbf{z}]$, say the $i_{1}$ th, which is a function distinct from all other diagonal elements.

Then, the $i_{1}$ th source of the mixture is identifiable in the following sense:
For any $N \times Q$ matrix $\mathbf{W}\left[z_{1}\right]$ such that the $N \times N$ combined channel-equalizer $z$-transform matrix $\mathbf{G}\left[z_{1}\right]=$ $\mathbf{W}\left[z_{1}\right] \mathbf{M}\left[z_{1}\right]$ is irreducible, if $\mathbf{W}\left[z_{1}\right] \boldsymbol{\Gamma}_{\mathbf{x}}\left[z_{1}\right] \mathbf{W}\left[z_{1}^{-1}\right]^{\mathrm{T}}$ and $\mathbf{W}\left[z_{1} z_{2}\right] \mathbf{C}_{\mathbf{x}}^{\mathbf{r}}[\mathbf{z}] \mathbf{W}\left[z_{1}^{-1}\right]^{\mathrm{T}}$ are both diagonal, then:

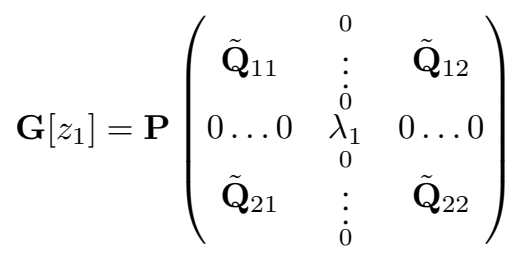

where $\lambda_{1} \neq 0$ is situated at the $i_{1}$ th row and $i_{1}$ th column and $\mathbf{P}$ is a permutation matrix.

Proof: One can write the assumptions of the above proposition as follows:

$$
\begin{gathered}
\mathbf{G}\left[z_{1}\right] \boldsymbol{\Gamma}_{\mathbf{s}}\left[z_{1}\right] \mathbf{G}\left[z_{1}^{-1}\right]^{\mathrm{T}}=\hat{\boldsymbol{\Gamma}}_{\mathbf{s}}\left[z_{1}\right] \\
\mathbf{G}\left[z_{1} z_{2}\right] \mathbf{C}_{\mathbf{s}}^{\mathbf{r}}[\mathbf{z}] \mathbf{G}\left[z_{1}^{-1}\right]^{\mathrm{T}}=\hat{\mathbf{C}}_{\mathbf{s}}^{\mathbf{r}}[\mathbf{z}]
\end{gathered}
$$

where $\hat{\boldsymbol{\Gamma}}_{\mathbf{s}}\left[z_{1}\right]$ and $\hat{\mathbf{C}}_{\mathbf{s}}^{\mathbf{r}}[\mathbf{z}]$ are diagonal matrices and $\hat{\boldsymbol{\Gamma}}_{\mathbf{s}}\left[z_{1}\right]$ satisfies the conditions associated to a power spectral $z$ transform matrix (that is ${ }^{1} \hat{\boldsymbol{\Gamma}}_{\mathbf{s}}\left[z_{1}\right]^{\mathrm{T}}=\hat{\boldsymbol{\Gamma}}_{\mathbf{s}}\left[z_{1}^{-1}\right]$ and $\hat{\boldsymbol{\Gamma}}_{\mathbf{s}}\left[e^{\imath \omega}\right] \geq$ $0, \forall \omega \in[0,2 \pi])$. Hence, following the proof in [11], one deduces from (17) that $\mathbf{G}\left[z_{1}\right]$ is a constant orthogonal matrix, say $\mathbf{G}\left[z_{1}\right]=\mathbf{Q}$ and (18) reads:

$$
\mathbf{Q C}_{\mathbf{s}}^{\mathbf{r}}[\mathbf{z}]=\hat{\mathbf{C}}_{\mathbf{s}}^{\mathbf{r}}[\mathbf{z}] \mathbf{Q}
$$

Denote by $Q_{m n}$ the $(m, n)$ th element of $\mathbf{Q}$ and by $C_{n}[\mathbf{z}]$ (resp. $\hat{C}_{n}[\mathbf{z}]$ ) the $n$th element of the diagonal matrix $\mathbf{C}_{\mathbf{s}}^{\mathbf{r}}[\mathbf{z}]$ (resp. $\left.\hat{\mathbf{C}}_{\mathbf{s}}^{\mathbf{r}}[\mathbf{z}]\right)$. In the above equation, the term at the $(m, n)$ th position reads $Q_{m n} C_{n}[\mathbf{z}]=\hat{C}_{m}[\mathbf{z}] Q_{m n}$. Because of the orthogonality, there is a non-zero element $Q_{\sigma\left(i_{1}\right) i_{1}}$ in the $i_{1}$ th column. Based on the assumption that $C_{i_{1}}[\mathbf{z}]$ is distinct from all other diagonal elements $C_{n}[\mathbf{z}], n \neq i_{1}$, one can obtain that on the same row $Q_{\sigma\left(i_{1}\right) n}=0$ if $n \neq i_{1}$. The orthogonality of $\mathbf{Q}$ then implies that in the $i_{1}$ th column, $Q_{n i_{1}}=0$ for $n \neq \sigma\left(i_{1}\right)$. This shows that the matrix $\mathbf{G}\left[z_{1}\right]$ has the desired structure.

One can notice that in Proposition 2, we have also that the matrix $\left(\begin{array}{cc}\tilde{\mathbf{Q}}_{11} & \tilde{\mathbf{Q}}_{12} \\ \tilde{\mathbf{Q}}_{21} & \tilde{\mathbf{Q}}_{22}\end{array}\right)$ is orthogonal. Additionally, two important comments should be made to link the above proposition with usual assumptions.

First, note that the assumption that $\boldsymbol{\Gamma}_{\mathbf{s}}\left[z_{1}\right]$ be diagonal is equivalent to the assumptions that the sources be uncorrelated. Similarly, the assumption that $\mathbf{C}_{\mathbf{s}}^{\mathbf{r}}[\mathbf{z}]$ is diagonal corresponds to the classical assumption that the sources be independent, although both are not equivalent. It has indeed been illustrated in [2] through several examples that mutual independence is a sufficient condition for separation, but is not always necessary. In the present formulation, we assume a weaker condition than independence. This allows us to deal with cases such where the sources are possibly spatially mutually dependent, such as in [2].

Consider also the situation where one or more diagonal elements of $\mathbf{C}_{\mathbf{s}}^{\mathbf{r}}[\mathbf{z}]$ vanish, say for example $C_{s_{i_{1}} s_{i_{1}}}^{\mathbf{r}}[\mathbf{z}]=0$ : in this case, and according to Assumption (ii) in Proposition 2 , the $i_{1}$-th source is identifiable only if there is no other zero element on the diagonal of $\mathbf{C}_{\mathbf{s}}^{\mathbf{r}}[\mathbf{z}]$. In Section V-B,

\footnotetext{
${ }^{1}$ Similarly to [11], only rational spectra are considered in the paper, that is $\boldsymbol{\Gamma}_{\mathbf{s}}\left[z_{1}\right]$ and $\hat{\boldsymbol{\Gamma}}_{\mathbf{s}}\left[z_{1}\right]$ have rational elements.
} 
it will be illustrated how in the case of i.i.d. signals, this condition implies the well-known necessary condition that at most one source may have vanishing $R$-th order cumulants. The condition given in Proposition 2 is hence more widely satisfied and weaker than the usual one: indeed an example has been given in [5] of non i.i.d. sources, which auto-cumulants with no delay vanish whereas the cross-cumulants at different times are non zero: our result confirm that a mixture of these sources can be separated.

\section{Identifiability of all sources}

We have shown in the previous section that it is possible to identify one source under a condition on the reference signal $\mathbf{r}(n)$. We now extend the result and explicit a condition to be satisfied so as to ensure that all sources can be identified. For that, we consider $N$ reference signals $\mathbf{r}_{i}(n), i=1, \ldots, N$ which may possibly be the same ones.

Proposition 3: Assume that there exist $N$ vector-valued reference signals $\mathbf{r}_{i}(n), i=1, \ldots, N$ and a permutation $\sigma$ of the set $\mathcal{I}_{N}$ such that the following condition hold:

A3. the matrices $\boldsymbol{\Gamma}_{\mathbf{s}}\left[z_{1}\right]$ and $\mathbf{C}_{\mathbf{s}}^{\mathbf{r}_{i}}[\mathbf{z}]$ for all $i \in \mathcal{I}_{N}$ are all diagonal,

A4. for all $i \in \mathcal{I}_{N}$ the $\sigma(i)$ th diagonal element of $\mathbf{C}_{\mathbf{s}}^{\mathbf{r}_{i}}[\mathbf{z}]$ is a function distinct from all other diagonal elements.

Then, the $N$ sources are identifiable up to a permutation and scaling factor in the following sense:

For any $N \times Q$ matrix $\mathbf{W}\left[z_{1}\right]$ such that the $N \times N$ combined channel-equalizer $z$-transform matrix $\mathbf{G}\left[z_{1}\right]=$ $\mathbf{W}\left[z_{1}\right] \mathbf{M}\left[z_{1}\right]$ is irreducible , if $\mathbf{W}\left[z_{1}\right] \boldsymbol{\Gamma}_{\mathbf{x}}\left[z_{1}\right] \mathbf{W}\left[z_{1}^{-1}\right]^{\mathrm{T}}$ and $\mathbf{W}\left[z_{1} z_{2}\right] \mathbf{C}_{\mathbf{x}}^{\mathbf{r}_{j}}[\mathbf{z}] \mathbf{W}\left[z_{1}^{-1}\right]^{\mathrm{T}}, j \in\{1, \ldots, N\}$ are all diagonal, then:

$$
\mathbf{G}\left[z_{1}\right]=\mathbf{P} \boldsymbol{\Lambda}
$$

where $\mathbf{P}$ is a permutation matrix and $\boldsymbol{\Lambda}$ is a diagonal matrix with non zero elements on the diagonal.

Proof: Using the same arguments as in the proof of Proposition 2 one obtains that $\mathbf{G}\left[z_{1}\right]=\mathbf{Q}$ is a constant orthogonal matrix. Under the assumptions of the proposition, we have in addition:

$$
\forall j \in\{1, \ldots, N\} \quad \mathbf{Q C}_{\mathbf{x}}^{\mathbf{r}_{j}}[\mathbf{z}]=\hat{\mathbf{C}}_{\mathbf{x}}^{\mathbf{r}_{j}}[\mathbf{z}] \mathbf{Q}
$$

Using arguments similar to the previous ones in the proof of Proposition 2, one obtains by induction that $\mathbf{G}\left[z_{1}\right]$ has the expected form: $\mathbf{G}\left[z_{1}\right]=\mathbf{P} \boldsymbol{\Lambda}$.

Let us make two remarks.

Remark 1: The reference signals $\mathbf{r}_{1}(n), \ldots, \mathbf{r}_{N}(n)$ may possibly be the same. In particular, if $\mathbf{r}_{1}(n)$ is such that the elements of $\mathbf{C}_{\mathbf{x}}^{\mathbf{r}_{1}}[\mathbf{z}]$ are mutually different, then one can consider $\mathbf{r}_{1}(n)=\mathbf{r}_{2}(n)=\ldots=\mathbf{r}_{N}(n)$ and the reference signals $\left\{\mathbf{r}_{1}(n), \ldots, \mathbf{r}_{N}(n)\right\}$ satisfy the condition of the proposition. In this case, $\mathbf{C}_{\mathbf{x}}^{\mathbf{r}_{1}}[\mathbf{z}]$ and $\boldsymbol{\Gamma}_{\mathbf{x}}\left[z_{1}\right]$ are enough to separate the sources. Similarly, each vector-valued reference signal $\mathbf{r}_{j}(n)$ (for $j$ fixed) is constituted of $R-2$ components, some or all of which may possibly be identical.

Remark 2: One could state a weaker condition instead of A4 and replace it with:

A $4^{\circ}$. for all $i \in \mathcal{I}_{N}$ the $\sigma(i)$ th element of the diagonal matrix $\mathbf{C}_{\mathbf{x}}^{\mathbf{r}_{i}}[\mathbf{z}]$ is a function distinct from the diagonal elements which indices are not in $\{\sigma(1), \ldots, \sigma(i-1)\}$. The condition $A 4^{\circ}$ can be easily interpreted: it says merely that the $\sigma(i)$ th source can be identified by using the reference $\mathbf{r}_{i}(n)$, and that the $\sigma(i)$ th source has not been identified previously by either one of the references $\mathbf{r}_{1}(n), \ldots, \mathbf{r}_{i-1}(n)$.

Finally, let us stress that Propositions 2 and 3 hold under the condition that $\mathbf{C}_{\mathbf{s}}^{\mathbf{r}}[\mathbf{z}]$ is diagonal: this corresponds in a weaker form to the independence of the sources. An interesting situation where this happens is detailed in the next Section.

\section{SEMI-BLIND REFERENCES}

The semi-blind context seems one of the most interesting and promising where a reference based approach can be used. Indeed, if a reference signal is available, one can obtain an effective separation method by using existing algorithms [7], [13]. A challenging issue consists in dealing with the validity of a given reference signal and in finding sufficient condition which can be used in a practical situation. We give here some elements only. Note also that in a blind context, reference signals can be constructed from the observations only [7], [13].

\section{A. Diagonal matrices of reference cumulants}

The conditions presented in section III require the sources be uncorrelated and $\mathbf{C}_{\mathbf{s}}^{\mathbf{r}}[\mathbf{z}]$ be diagonal. We now give sufficient conditions on the vector-valued reference signal under which it may be used for separation. This gives a more accurate view of the results presented in [7]. We have the following proposition:

Proposition 4: For $i \in \mathcal{I}_{N}$, consider $N$ signals $\tilde{s}_{i}(n)$ and denote $\check{s}_{i}(n) \triangleq\left(s_{i}(n), \tilde{s}_{i}(n)\right)$. Assume that:

A5. there exist a $(R-2) \times N$ filter with impulse response $\mathbf{T}(n)$ such that:

$$
\mathbf{r}(n)=\sum_{k \in \mathbb{Z}} \mathbf{T}(k) \tilde{\mathbf{s}}(n-k)
$$

A6. the processes $\check{s}_{i}(n)$ are mutually independent for $i \in \mathcal{I}_{N}$. Then, the matrix $\mathbf{C}_{\mathbf{s}}^{\mathbf{r}}[\mathbf{z}]$ is diagonal.

Proof: For all $j \in \mathcal{I}_{R-2}$, we have $r_{j}(n)=$ $\sum_{p \in \mathcal{I}_{N}} \sum_{k \in \mathbb{Z}} T_{j p}(k) \tilde{s_{p}}(n-k)$. Replacing $r_{j}\left(n-n_{2}\right)$ for all $j$ in (4) and then using multilinearity, one can write:

$$
\begin{aligned}
C_{s_{i_{1}} s_{i_{2}}}^{\mathbf{r}}(\mathbf{n})=\sum_{\mathbf{p} \in \mathcal{I}_{N}^{R-2}} \sum_{\mathbf{k} \in \mathbb{Z}^{R-2}}\left(\prod_{j=1}^{R-2} T_{j p_{j}}\left(k_{j}\right)\right) \times \\
\operatorname{Cum}\left\{s_{i_{1}}(n), s_{i_{2}}\left(n-n_{1}\right), \tilde{s}_{p_{1}}\left(n-n_{2}-k_{1}\right), \ldots,\right. \\
\left.\tilde{s}_{p_{R-2}}\left(n-n_{2}-k_{R-2}\right)\right\}
\end{aligned}
$$

By the independence assumptions on the sources $A 6$ and on the signals $\tilde{s}_{i}(n), i \in \mathcal{I}_{N}$, the above cumulant expressions systematically vanish when $i_{1} \neq i_{2}$. It follows by (5) that $\mathbf{C}_{\mathbf{s}}^{\mathbf{r}}[\mathbf{z}]$ is diagonal.

A specific but interesting situation where the condition of the above Proposition is satisfied is when for all $i, \tilde{s}_{i}(n)=$ $s_{i}(n)$. In other words, the references are obtained from the sources by a MIMO filtering operation. This is the case 
considered in [7], and also in [13]. In particular, the observations being themselves obtained by a MIMO filtering of the sources, they can serve as reference signal upon the additional assumptions which are specified before in Propositions 2 and 3.

Another specific situation where the condition of the above Proposition are satisfied is when $\mathbf{r}(n)$ depends on one source (say the $i_{1}$-th) and is independent of the other ones. In this case, one can observe additionally that $\mathbf{C}_{\mathbf{s}}^{\mathbf{r}}[\mathbf{z}]$ contains a single non zero element: this seemingly very favorable assumption could more likely correspond to the situation where additional extra information is available which can be used to extract the $i_{1}$-th source. This semi-blind context is considered in simulations to illustrate the potentialities of our results.

\section{B. Simulations}

We now illustrate by simulations the validity of the results in the previous section. We assume that some information is partially available on the sources through the reference signal: we assume the reference is a binary signal which depends on the sign of one source signal in a non deterministic way: such a situation could be of interest (e.g. in a telecommunication context) and, to the best of our knowledge, it has not been considered anywhere before. Another example has been treated in [3] where a partial information is available on the phase or the modulus of a complex-valued source signal.

More precisely, we deal with the case where $\tilde{s}_{i}(n)=$ $\operatorname{sign}\left(s_{i}(n)\right) \epsilon_{i}(n)$ where for all $i, \epsilon_{i}(n)$ is a binary i.i.d. Bernouilli process with $P\left(\epsilon_{i}(n)=1\right)=p$ and $P\left(\epsilon_{i}(n)=\right.$ $-1)=1-p$. The processes $\epsilon_{i}(n)$ are mutually independent and independent of the sources. The signals $\tilde{s}_{i}(n)$ then satisfy the conditions of Proposition 4 and each $\tilde{s}_{i}(n)$ contains information on the sign of $s_{i}(n): s_{i}(n)$ and $\tilde{s}_{i}(n)$ indeed have the same sign with probability given by the parameter $p$.

In our simulations, we tried to extract one particular source (say the $i_{1}$ th) and we considered the reference signal defined by $r(n)=\sum_{k} t_{i_{1}}(k) \tilde{s}_{i_{1}}(n-k)$ where $\left(t_{i_{1}}(k)\right), k \in \mathbb{Z}$ is the impulse response of a scalar filter. We considered for $\left(t_{i_{1}}(k)\right), k \in \mathbb{Z}$ both the identity filter and a randomly driven FIR filter of length 3. $r(n)$ thus contains directly or indirectly information on the sign of $s_{i}(n)$ and it can be seen that only the $i_{1}$ th diagonal element of the corresponding matrix $\mathbf{C}_{\mathbf{s}}^{\mathbf{r}}[z]$ is non zero.

A set of $N$ mutually independent and temporally i.i.d. uniformly distributed sources have been generated. They have been mixed by a $Q \times N$ randomly chosen FIR filter of length 3 . We then used the real valued fourth order cumulant-based contrast proposed in [7] and the associated algorithm to test the effectiveness of our result. Different values of the parameter $p$ and different sample sizes have been considered. Results obtained with 1000 Monte-Carlo realizations are reported in Table I for the values $N=3, Q=4$ and in Table II for $N=4, Q=5 .:$ the averaged value and the $90 \%$ quantile value of the the mean square error on the estimated source are reported.

One can observe the effectiveness of the separation when $p \neq 0.5$ : it should indeed be no surprise that no separation is obtained for $p=0.5$ since in this case, $\tilde{s}_{i}(n)$ is independent of $s_{i}(n)$, implying that the matrix $\mathbf{C}_{\mathbf{s}}^{\mathbf{r}}[z]$ is identically zero. On the contrary, $p \neq 0.5$ implies that $\left(s_{i_{1}}(n), \tilde{s}_{i_{1}}(n)\right)$ are dependent, but independent of all other sources. In such considered cases, this yields a sufficient condition on the reference signals so that the methods [7], [13] can be used.

\section{LINK WITH FORMER RESULTS}

\section{A. Second-order identifiability condition}

The results presented in the previous sections are connected with the results in [11] on second-order identifiability of FIRMIMO systems. In the case where $R=2$ and no reference signal is considered, the Propositions 2 and 3 lead to the result in [11]. The role played by $\boldsymbol{\Gamma}\left[z_{1}\right]$ and $\mathbf{C}_{\mathbf{s}}^{\mathbf{r}}[\mathbf{z}]$ in Proposition 3 is played simultaneously by the power spectral matrix $\boldsymbol{\Gamma}\left[z_{1}\right]$ in [11]. More precisely, it is seen from the proof that using the second-order statistics only (that is the spectral matrix), the FIR-MIMO system may be identified up to an orthogonal transform. The remaining orthogonal transform is identified thanks to a diversity which can be satisfied either at second order (as in [11]) or at $R$-th order for a given set of reference signals (as in our Propositions 2 and 3). In other words, the use of reference signals allows one to consider another interesting signal diversity. It follows that our identifiability condition is actually much weaker than the usual known one, since the diversity condition may be satisfied at any given statistic order.

\section{B. i.i.d. sources}

We now show that the identifiability condition in Proposition 2 is necessarily satisfied in the results given by [7] and in [13]. The case of non i.i.d. sources, which is treated in [7] but not in [13], is addressed in Section V-C. Both works focus mainly on the problem of extracting one source in the context where the reference signals are implicitly obtained by a filtering operation on the sources. In particular, the conditions of Proposition 4 hold true, which implies $C_{s_{i_{1}} s_{i_{2}}}^{\mathbf{r}}(\mathbf{n})=0$ when $i_{1} \neq i_{2}$. More precisely, we have $\tilde{\mathbf{s}}(n)=\mathbf{s}(n)$ and hence we can write:

$$
\forall j \in \mathcal{I}_{R-2} \quad r_{j}(n)=\sum_{p \in \mathcal{I}_{N}} \sum_{l \in \mathbb{Z}} T_{j p}(l) s_{p}(n-l)
$$

Assuming in addition that the sources are temporally i.i.d. we obtain:

$$
C_{s_{i} s_{i}}^{\mathbf{r}}(\mathbf{n})= \begin{cases}0 & \text { if } n_{1} \neq 0 \\ \left(\prod_{j=1}^{R-2} T_{j i}\left(-n_{2}\right)\right) \mathcal{K}_{s_{i}}^{(R)} & \text { otherwise }\end{cases}
$$

where $\mathcal{K}_{s_{i}}^{(R)}$ denotes the $R$-th order auto-cumulant $\operatorname{Cum}\left\{s_{i}(n), \ldots, s_{i}(n)\right\}$, which does not depend on $n$ because of stationarity (assumption A0). It follows that

$$
\begin{aligned}
C_{s_{i} s_{i}}^{\mathbf{r}}[\mathbf{z}]= & \sum_{n_{2} \in \mathbb{Z}} C_{s_{i} s_{i}}^{\mathbf{r}}\left(0, n_{2}\right) z_{2}^{-n_{2}} \\
& =\mathcal{K}_{s_{i}}^{(R)} \sum_{n_{2} \in \mathbb{Z}}\left[\left(\prod_{j=1}^{R-2} T_{j i}\left(-n_{2}\right)\right) z_{2}^{-n_{2}}\right] .
\end{aligned}
$$




\begin{tabular}{|l|c||cccc|cccc|}
\cline { 2 - 9 } \multicolumn{1}{c|}{} & \multicolumn{1}{c|}{ Number of } & \multicolumn{4}{c|}{$\left(t_{i_{1}}(n)\right) \leftrightarrow$ identity } & \multicolumn{4}{c|}{$\left(t_{i_{1}}(n)\right) \leftrightarrow$ FIR random } \\
\cline { 2 - 10 } \multicolumn{1}{c|}{} & samples & $p=1$ & $p=0.9$ & $p=0.7$ & $p=0.5$ & $p=1$ & $p=0.9$ & $p=0.7$ & $p=0.5$ \\
\hline \multirow{2}{*}{ Average } & 1000 & 0.0078 & 0.0249 & 0.1548 & 1.0780 & 0.0379 & 0.0945 & 0.7079 & 1.1340 \\
value & 5000 & 0.0015 & 0.0050 & 0.0331 & 1.0799 & 0.0130 & 0.0248 & 0.2470 & 1.1303 \\
& 10000 & $7.57 \mathrm{e}-4$ & 0.0025 & 0.0168 & 1.0762 & 0.0073 & 0.0142 & 0.1383 & 1.1335 \\
\hline \multirow{2}{*}{$90 \%$} & 1000 & 0.0109 & 0.0345 & 0.2197 & 1.2415 & 0.0770 & 0.2186 & 1.1956 & 1.2653 \\
quantile & 5000 & 0.0022 & 0.0069 & 0.0466 & 1.2393 & 0.0151 & 0.0424 & 0.5853 & 1.2585 \\
& 10000 & 0.0010 & 0.0035 & 0.0238 & 1.2400 & 0.0062 & 0.0190 & 0.3369 & 1.2647 \\
\hline
\end{tabular}

TABLE I

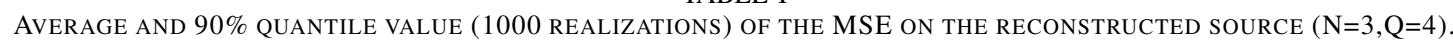

\begin{tabular}{|l|c||cccc|cccc|}
\cline { 2 - 9 } \multicolumn{1}{c|}{} & Number of & \multicolumn{4}{c|}{$\left(t_{i_{1}}(n)\right) \leftrightarrow$ identity } & \multicolumn{3}{c|}{$\left(t_{i_{1}}(n)\right) \leftrightarrow$ FIR random } \\
\cline { 2 - 9 } \multicolumn{1}{c|}{} & samples & $p=1$ & $p=0.9$ & $p=0.7$ & $p=0.5$ & $p=1$ & $p=0.9$ & $p=0.7$ & $p=0.5$ \\
\hline \multirow{2}{*}{ Average } & 1000 & 0.0130 & 0.0416 & 0.2403 & 1.2262 & 0.0498 & 0.1338 & 0.9502 & 1.2206 \\
value & 5000 & 0.0026 & 0.0084 & 0.0545 & 1.2290 & 0.0148 & 0.0330 & 0.3938 & 1.1077 \\
& 10000 & 0.0013 & 0.0042 & 0.0280 & 1.2298 & 0.0104 & 0.0234 & 0.2285 & 1.0544 \\
\hline \multirow{2}{*}{$90 \%$} & 1000 & 0.0169 & 0.0547 & 0.3181 & 1.3586 & 0.0859 & 0.2562 & 1.3550 & 1.3637 \\
quantile & 5000 & 0.0034 & 0.0110 & 0.0717 & 1.3548 & 0.0156 & 0.0512 & 0.9503 & 1.3513 \\
& 10000 & 0.0017 & 0.0055 & 0.0365 & 1.3595 & 0.0093 & 0.0333 & 0.5211 & 1.3622 \\
\hline
\end{tabular}

TABLE II

Average And 90\% Quantile Value (1000 Realizations) OF THE MSE on the ReCONSTRUCted Source $(\mathrm{N}=4, \mathrm{Q}=5)$.

a) Link with [7]: Define

$$
\mathcal{C}_{R}^{\max } \triangleq \max _{i=1}^{N} \sup _{k \in \mathbb{Z}}\left|C_{s_{i} s_{i}}^{\mathbf{r}}(0, k)\right|
$$

Assume as in [7] that the above supremum is finite and uniquely reached, that is

$$
\begin{aligned}
\left|C_{s_{i_{1}} s_{i_{1}}}^{\mathbf{r}}\left(0, k_{1}\right)\right| & =\mathcal{C}_{R}^{\max }<\infty \text { and: } \\
\forall(i, k) & \neq\left(i_{1}, k_{1}\right) \quad\left|C_{s_{i} s_{i}}^{\mathbf{r}}(0, k)\right|<\mathcal{C}_{R}^{\max } .
\end{aligned}
$$

Considering (25) one can then see that for $i \neq i_{1}, C_{s_{i} s_{i}}^{\mathbf{r}}[\mathbf{z}]$ and $C_{s_{i_{1}} s_{i_{1}}}^{\mathbf{r}}[\mathbf{z}]$ are distinct functions. The source $s_{i_{1}}(n)$ is hence identifiable according to Proposition 2. This is confirmed by the results in [7] which show the validity of a particular contrast function. The validity condition for the contrast function in [7] is hence a much stronger condition than the identifiability condition which is given here.

Having a closer look at Equation (25), one can distinguish the importance of the identifiability conditions at two distinct levels. On the one hand, the cumulant values play a fundamental role: indeed, the source $i_{1}$ can be extracted if and only if the diagonal element $C_{s_{i_{1}} s_{i_{1}}}^{\mathbf{r}}[\mathbf{z}]$ of $\mathbf{C}_{\mathbf{s}}^{\mathbf{r}}[\mathbf{z}]$ is distinct from the others. From (25), this necessarily implies that either $\mathcal{K}_{s_{i_{1}}}^{(R)}$ be non zero, or that source $i_{1}$ be the only source with vanishing $R$-th order cumulant. This corresponds to the wellknown condition that at most one source may be Gaussian. On the other hand, as soon as $\mathcal{K}_{s_{i_{1}}}^{(R)} \neq 0$, there always exist an appropriate choice of the reference system in (25) which makes possible the separation of the corresponding source.

b) Link with [13]: In [13], fourth-order cumulants $(R=$ 4) are considered and it is assumed that the reference $\mathbf{r}(n)$ consists of two identical components $r_{1}(n)=r_{2}(n) \triangleq r(n)$, where $r(n)$ is written as

$$
r(n)=\sum_{p \in \mathcal{I}_{N}} \sum_{l \in \mathbb{Z}} t_{p}(l) s_{p}(n-l)
$$

Then, one can easily obtain:

$$
C_{s_{i} s_{i}}^{\mathbf{r}}[\mathbf{z}]=\mathcal{K}_{s_{i}}^{(4)} \sum_{n_{2} \in \mathbb{Z}} t_{i}\left(-n_{2}\right)^{2} z_{2}^{-n_{2}}
$$

It is shown in [13] that if all the values $\lambda_{i}(k) \triangleq\left|t_{i}(k)\right|^{2} \mathcal{K}_{s_{i}}^{(4)}$ are distinct for $i \in \mathcal{I}_{N}$ and $k \in \mathbb{Z}$, then the corresponding source can be recovered. Clearly, distinct values of $\lambda_{i}(k)$ correspond to distinct rational functions $C_{s_{i} s_{i}}^{\mathbf{r}}[\mathbf{z}]$. Proposition 2 and 3 hence show that the separating system can be identified under conditions weaker than those in [13]. It is sufficient that there exist $N$ choices of $\mathbf{r}(n)$ such that the functions in (25) (or (29)) are different for different $i$. This seems quite a weak condition on the reference system $(\mathbf{T}(k))_{k \in \mathbb{Z}}$. From (29), it is seen in addition that the condition on the reference system does not depend on the particular values of the auto-cumulants of the sources, if different from zero.

\section{C. non i.i.d. sources}

We now move to the case of non i.i.d. sources. This case has been considered in [7]. Let us show that the validity conditions in [7] imply that the identifiability condition given in this paper is satisfied. Define $y_{i}(n)$ as the result of a scalar filter applied on $s_{i}(n)$. If $g_{i}\left[z_{1}\right]$ is the corresponding $z$-transform of the scalar filter, we have according to (7):

$$
C_{y_{i} y_{i}}^{\mathbf{r}}[\mathbf{z}]=g_{i}\left[z_{1}^{-1}\right] g_{i}\left[z_{1} z_{2}\right] C_{s_{i} s_{i}}^{\mathbf{r}}[\mathbf{z}]
$$

In [7], the quantity $\left|C_{y_{i} y_{i}}^{\mathbf{r}}(0,0)\right|$ is considered when $y_{i}(n)$ is the particular output corresponding to the maximum value of this quantity over a set of unit-norm filters (the supremum are assumed to be reached and finite). The validity condition in [7] expresses that there should be an index (say the index $i_{1}$ ) such that $\left|C_{y_{i_{1}} y_{i_{1}}}^{\mathbf{r}}(0,0)\right|>\left|C_{y_{i} y_{i}}^{\mathbf{r}}(0,0)\right|$ for all $i \neq i_{1}$. In this case precisely, we must have $C_{y_{i_{1}} y_{i_{1}}}^{\mathbf{r}}[\mathbf{z}] \neq C_{y_{i} y_{i}}^{\mathbf{r}}[\mathbf{z}]$ and thus $C_{s_{i_{1} s_{i_{1}}}}^{\mathbf{r}}[\mathbf{z}] \neq C_{s_{i} s_{i}}^{\mathbf{r}}[\mathbf{z}]$ according to (30). Again, Proposition 2 hence provides weaker identifiability conditions than the results in [7].

\section{CONCLUSION}

General identifiability conditions of a separating system have been given by considering reference signals in higher order statistics. In particular, our new results include former 
existing ones. Known results based on second-order statistics have been generalized to higher-order statistics and we have shown that the given identifiability conditions are weaker than the assumptions required in former papers in which referencebased approaches have been considered. The interest of our approach has been clearly illustrated in a semi-blind context. These new results, although theoretical ones, open up the possibility of developing novel algorithms.

\section{REFERENCES}

[1] M. Castella, P. Bianchi, A. Chevreuil, and J.-C. Pesquet. A blind source separation framework for detecting CPM sources mixed by a convolutive MIMO filter. Signal Processing, 86(8):1950-1967, August 2007.

[2] M. Castella and P. Comon. Blind separation of instantaneous mixtures of dependent sources. In Proc. of ICA'07, volume 4666 of $L N C S$, pages 9-16, London, UK, September 2007.

[3] M. Castella and E. Moreau. Reference based contrast functions in a semi-blind context. In Proc. of ICA'09, Paraty-RJ, Brazil, March 2009. Accepted for publication.

[4] M. Castella, E. Moreau, and J.-C. Pesquet. A quadratic MISO contrast function for blind equalization. In Proc. IEEE Int. Conf. on Acoustics, Speech and Signal Processing (ICASSP), pages 681-684, Montréal, Canada, May 2004.

[5] M. Castella, J.-C. Pesquet, and A. P. Petropulu. A family of frequencyand time-domain contrasts for blind separation of convolutive mixtures of temporally dependent signals. IEEE Trans. Signal Processing, 53(1):107-120, January 2005.

[6] M. Castella, S. Rhioui, E. Moreau, and J.-C. Pesquet. Source separation by quadratic contrast functions: a blind approach based on any higherorder statistics. In Proc. IEEE Int. Conf. on Acoustics, Speech and Signal Processing (ICASSP), Philadelphia, USA, March 2005.

[7] M. Castella, S. Rhioui, E. Moreau, and J.-C. Pesquet. Quadratic higherorder criteria for iterative blind separation of a MIMO convolutive mixture of sources. IEEE Trans. Signal Processing, 55(1):218-232, January 2007.

[8] P. Comon. Independent component analysis, a new concept. Signal Processing, 36(3):287-314, April 1994.

[9] P. Comon. Contrasts for multichannel blind deconvolution. IEEE Signal Processing Letters, 3(7):209-211, July 1996.

[10] A. Gorokhov and P. Loubaton. Subspace based techniques for blind separation of convolutive mixtures with temporally correlated sources. IEEE Trans. Circuits and Systems I, 44(9):813-820, September 1997.

[11] Y. Hua and J. K. Tugnait. Blind identifiability of FIR-MIMO systems with colored input using second order statistics. IEEE Signal Processing Letters, 7(12):348-350, December 2000.

[12] M. Kawamoto, K. Kohno, and Y. Inouye. Eigenvector algorithms using reference signals. In Proc. IEEE Int. Conf. on Acoustics, Speech and Signal Processing (ICASSP), volume 5, May 2006. Digital Object Identifier 10.1109/ICASSP.2006.1661407.

[13] M. Kawamoto, K. Kohno, and Y. Inouye. Eigenvector algorithms incorporated with reference systems for solving blind deconvolution of MIMO-IIR linear systems. IEEE Signal Processing Letters, 14(12):996999, December 2007.

[14] E. Moreau and J.-C. Pesquet. Generalized contrasts for multichanel blind deconvolution of linear systems. IEEE Signal Processing Letters, 4(6):182-183, June 1997.

[15] E. Moreau, J.-C. Pesquet, and N. Thirion-Moreau. Convolutive blind signal separation based on asymmetrical contrast functions. IEEE Trans. Signal Processing, 55(1):356-371, January 2007.

[16] C. Simon, P. Loubaton, and C. Jutten. Separation of a class of convolutive mixtures: a contrast function approach. Signal Processing, (81):883-887, 2001.

[17] L. Tong, Y. R. Liu, V. Soon, and Y. Huang. Indeterminacy and identifiability of blind identification. IEEE Trans. Circuits Syst., 38:499509, May 1991.

[18] J. K. Tugnait. Identification and deconvolution of multichannel linear non-gaussian processes using higher order statistics and inverse filter criteria. IEEE Trans. Signal Processing, 45(3):658-672, March 1997.

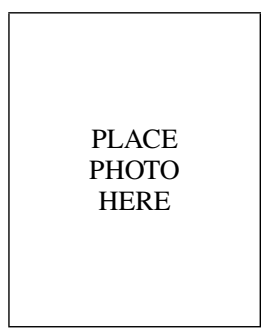

Marc Castella was born in 1976 in Courbevoie, France. In 2000, he received the "Agrégation" degree in the field of applied physics and in 2001 he received the M.Sc. degree in electrical engineering, from both the "École Normale Supérieure de Cachan" and the "Université Paris-Sud, Orsay" (France). He obtained his Ph.D. degree from "Université de Marne-la-Vallée" (France) in 2004. Since then, he has been "Maître de Conférence" in Evry (France) at "Télécom \& Management SudParis" (formerly "Institut National des Télécommunications (INT)"). He is also a member of the UMR-CNRS 5157 research team SAMOVAR. His research activities have focused on the problem blind source separation in general.

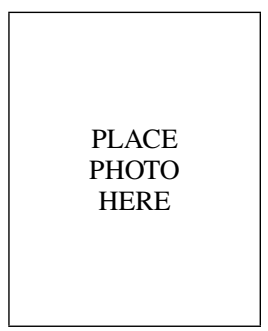

Eric Moreau was born in Lille, France. He graduated from the "Ecole Nationale Supérieure des Arts et Métiers" (ENSAM), Paris, France, in 1989 and received the "Agrégation de Physique" degree from the "Ecole Normale Supérieure de Cachan" in 1990. He received the DEA degree in 1991 and the Ph.D. degree in 1995, both in the field of signal processing and from the University of Paris-Sud, France. From 1995 to 2001, he was assistant professor within the Telecommunications Department of the Engineering School "Institut des Sciences de l'Ingénieur de Toulon et du Var" (ISITV), La Valette, France. He is currently Professor at University of Toulon, France. His main research interests are in statistical signal processing using high-order statistics. 